\title{
Some monotonicity properties in F-normed Musielak-Orlicz spaces
}

\author{
RADOSŁAW KACZMAREK(D)
}

To my supervisor, Prof. Henryk Hudzik.

\begin{abstract}
Strict monotonicity, lower local uniform monotonicity, upper local uniform monotonicity and their orthogonal counterparts are considered in the case of Musielak-Orlicz function spaces $L^{\Phi}(\mu)$ endowed with the Mazur-Orlicz F-norm as well as in the case of their subspaces $E^{\Phi}(\mu)$ with the F-norm induced from $L^{\Phi}(\mu)$. The presented results generalize some of the results from Cui et al. (Aequ Math 93:311-343, 2019) and Hudzik et al. (J Nonlinear Convex Anal 17(10):1985-2011, 2016), obtained only for Orlicz spaces as well as their subspaces of order continuous elements equipped with the Mazur-Orlicz F-norm.
\end{abstract}

Mathematics Subject Classification. Primary 46E30; Secondary 46A80.

Keywords. Musielak-Orlicz spaces, Mazur-Orlicz F-norm, F-normed Köthe spaces, Strict monotonicity, Orthogonal strict monotonicity, Lower local uniform monotonicity, Orthogonal lower local uniform monotonicity, Upper local uniform monotonicity, Orthogonal upper local uniform monotonicity.

\section{Introduction and preliminaries}

Let us denote by $\mathbb{N}, \mathbb{R}$ and $\mathbb{R}_{+}$the sets of natural, real and nonnegative real numbers, respectively.

Given any real vector space $X$, the functional $X \ni x \rightarrow\|x\| \in \mathbb{R}_{+}:=[0, \infty)$, is called an $F$-norm if the following conditions are satisfied:

(i) $\|x\|=0$ if and only if $x=0$,

(ii) $\|-x\|=\|x\|$ for all $x \in X$,

(iii) $\|x+y\| \leq\|x\|+\|y\|$ for all $x, y \in X$,

(iv) $\left\|\lambda_{n} x_{n}-\lambda x\right\| \rightarrow 0$ whenever $\left\|x_{n}-x\right\| \rightarrow 0$ and $\lambda_{n} \rightarrow \lambda$ for any $x \in X$, $\left(x_{n}\right)_{n=1}^{\infty}$ in $X, \lambda \in \mathbb{R}$ and $\left(\lambda_{n}\right)_{n=1}^{\infty}$ in $\mathbb{R}$. 
An $F$-normed space $X=(X,\|\cdot\|)$ is said to be an $F$-space if it is complete with respect to the $F$-norm topology. If a lattice $X$ is endowed with a monotone $F$-norm $\|$. $\|$ (i.e., the condition $|y| \leq|x|$ implies $\|y\| \leq\|x\|$ for $x, y \in X$ ), under which $X$ is topologically complete, then $X=(X,\|\cdot\|)$ is said to be an $F$-lattice.

The notation $S(X)$ (resp. $B(X))$ stands for the unit sphere (resp. the closed unit ball) of a real $F$-normed space $(X,\|\cdot\|)$.

In the whole paper, we will assume that $(\Omega, \Sigma, \mu)$ is a complete $\sigma$-finite and non-atomic measure space. Let us denote by $L^{0}(\mu)=L^{0}(\Omega, \Sigma, \mu)$ the space of all (equivalence classes of) real-valued and $\Sigma$-measurable functions on $\Omega$.

Definition 1.1. An $F$-space $\left(E,\|\cdot\|_{E}\right)$ is called an $F$-normed Köthe space if it is a linear subspace of $L^{0}$ satisfying the following condition:

if $x \in L^{0}, y \in E$ and $|x| \leq|y| \mu-a . e$. , then $x \in E$ and $\|x\|_{E} \leq\|y\|_{E}$.

Denote by $E_{+}$the positive cone of $E$, that is, $E_{+}=\{x \in E: x \geq 0\}$.

Let us recall now the definitions of the necessary monotonicity properties.

Definition 1.2. An $F$-normed Köthe space $\left(E,\|\cdot\|_{E}\right)$ is said to be strictly monotone $(E \in(S M)$ for short) if for any $x, y \in E$ such that $0 \leq y \leq x$, we have $\|y\|_{E}<\|x\|_{E}$ whenever $y \neq x$ (or equivalently $\|x-y\|_{E}<\|x\|_{E}$ whenever $0 \leq y \leq x$ and $y \neq 0$ ) (see [1]).

Definition 1.3. An $F$-normed Köthe space $E$ is said to be orthogonally strictly monotone $\left(E \in(O S M)\right.$ for short) if for any $x \in E_{+} \backslash\{0\}$ and any $A \in \Sigma \cap$ $\operatorname{supp} x$ with $\mu(A)>0$, we have $\left\|x \chi_{\Omega \backslash A}\right\|_{E}<\|x\|_{E}$.

Definition 1.4. An $F$-normed Köthe space $\left(E,\|\cdot\|_{E}\right)$ is said to be lower locally uniformly monotone $\left(E \in(L L U M)\right.$ for short) if for any $x \in E$ and $\left(x_{n}\right)_{n=1}^{\infty}$ in $E$ such that $0 \leq x_{n} \leq x$ for all $n \in \mathbb{N}$ and $\left\|x_{n}\right\|_{E} \rightarrow\|x\|_{E}$ as $n \rightarrow \infty$, the condition $\left\|x-x_{n}\right\|_{E} \rightarrow 0$ as $n \rightarrow \infty$ holds.

Definition 1.5. An $F$-normed Köthe space $\left(E,\|\cdot\|_{E}\right)$ is said to be orthogonally lower locally uniformly monotone $(E \in(O L L U M)$ for short) if for any $x \in$ $E_{+} \backslash\{0\}$ and any $\left(A_{n}\right)_{n=1}^{\infty}$ in $\Sigma$ the following implication is satisfied:

$$
\left(\left\|x \chi_{A_{n}}\right\|_{E} \underset{n \rightarrow \infty}{\rightarrow}\|x\|_{E}\right) \Rightarrow\left(\left\|x-x \chi_{A_{n}}\right\|_{E}=\left\|x \chi_{\Omega \backslash A_{n}}\right\|_{E} \underset{n \rightarrow \infty}{\rightarrow} 0\right) .
$$

Definition 1.6. An $F$-normed Köthe space $\left(E,\|\cdot\|_{E}\right)$ is said to be upper locally uniformly monotone $\left(E \in(U L U M)\right.$ for short) if for any $x \in E_{+}$and $\left(x_{n}\right)_{n=1}^{\infty}$ in $E_{+}$such that $x \leq x_{n}$ for all $n \in \mathbb{N}$ and $\left\|x_{n}\right\|_{E} \rightarrow\|x\|_{E}$ as $n \rightarrow \infty$, the condition $\left\|x_{n}-x\right\|_{E} \rightarrow 0$ as $n \rightarrow \infty$ holds.

Definition 1.7. An F-normed Köthe space $\left(E,\|\cdot\|_{E}\right)$ is said to be orthogonally upper locally uniformly monotone $(E \in(O U L U M)$ for short) if for any $x \in$ $E_{+} \backslash\{0\}$ such that $\mu(\operatorname{supp} E \backslash \operatorname{supp} x)>0$ and any $x_{n} \in E_{+}$with $\operatorname{supp} x_{n} \subset$ $\operatorname{supp} E \backslash \operatorname{supp} x$, if $\left\|x+x_{n}\right\|_{E} \rightarrow\|x\|_{E}$ then $\left\|x_{n}\right\|_{E} \rightarrow 0$ as $n \rightarrow \infty$. 
A function $\Phi: \Omega \times[0, \infty) \rightarrow[0, \infty]$ is said to be a monotone Musielak-Orlicz function if $\Phi(t, 0)=0, \Phi(t,$.$) is non-decreasing and continuous on \left[0, b_{\Phi}(t)\right)$ for $\mu$-a.e. $t \in \Omega$, where

$$
b_{\Phi}(t):=\sup \{u \geq 0: \Phi(t, u)<\infty\},
$$

left continuous at $b_{\Phi}(t)$, i.e. $\lim _{u \rightarrow b_{\Phi}(t)_{-}} \Phi(t, u)=\Phi\left(t, b_{\Phi}(t)\right) \in(0, \infty]$ for $\mu$-a.e. $t \in \Omega, \lim _{u \rightarrow \infty} \Phi(t, u)>0$ for $\mu$-a.e. $t \in \Omega$ and $\Phi(t, u)$ is $\Sigma$-measurable for all $u \in \mathbb{R}_{+}$.

Let us also define

$$
a_{\Phi}(t):=\sup \{u \geq 0: \Phi(t, u)=0\}
$$

and denote:

$$
\begin{aligned}
\Omega_{o c} & =\left\{t \in \Omega: b_{\Phi}(t)=\infty\right\}, \\
\operatorname{supp} a_{\Phi} & =\left\{t \in \Omega: a_{\Phi}(t)>0\right\} \text { and } \\
\Omega_{n s i} & =\left\{t \in \Omega: \Phi(t, .) \text { is not strictly increasing on } \mathbb{R}_{+}\right\} .
\end{aligned}
$$

The functions $a_{\Phi}($.$) and b_{\Phi}($.$) are \Sigma$-measurable. The proof of this fact goes in the same way as the proof of Proposition 5.1 from [2], p. 175 (see also the earlier proof in [4]), where only the fact that for $\mu$-a.e. $t \in \Omega$, the function $\Phi(t, u)$ is continuous on $[0, \infty)$ with respect to $u$ was important (it is known that the convexity of such function implies its continuity on the interval, where its values are finite except for the right hand side point of the interval).

We say that a monotone Musielak-Orlicz function $\Phi$ satisfies the $\Delta_{2}$ condition (resp. the $\Delta_{2}\left(\Omega_{o c}\right)$-condition) if there exist a set $\Omega_{1} \in \Sigma$ with $\mu\left(\Omega_{1}\right)=0$, a constant $K>0$ and a function $0 \leq h \in L^{1}(\Omega, \Sigma, \mu)$ (resp. a function $\left.0 \leq h \in L^{1}\left(\Omega_{o c}, \Sigma, \mu\right)\right)$ such that $\Phi(t, 2 u) \leq K \Phi(t, u)+h(t)$ for all $t \in \Omega \backslash \Omega_{1}$ (resp. for all $t \in \Omega_{o c} \backslash \Omega_{1}$ ) and any $u \geq 0$. We will write then shortly that $\Phi \in \Delta_{2}$ (resp. $\Phi \in \Delta_{2}\left(\Omega_{o c}\right)$ ). It is easy to see that the following two conditions are equivalent:

1. $\Phi \in \Delta_{2}$,

2. there exist a set $\Omega_{1} \in \Sigma$ with $\mu\left(\Omega_{1}\right)=0$, a constant $K \geq 1$ and a measurable function $\delta \geq 0$ with $\Phi(t, \delta(t)) \in L^{1}(\Omega, \Sigma, \mu)$ such that $\Phi(t, 2 u) \leq K \Phi(t, u)$ for all $t \in \Omega \backslash \Omega_{1}$ and any $u \geq \delta(t)$.

Note that if $\Phi \in \Delta_{2}$, then $\Phi(t$,.) has finite values only for $\mu$-a.e. $t \in \Omega$ (the proof is the same as the proof of Proposition 5.3 in [2]).

For any monotone Musielak-Orlicz function $\Phi$, let us determine the functional $I_{\Phi}: L^{0}(\mu) \rightarrow[0, \infty]$ defined by the formula $I_{\Phi}(x)=\int_{\Omega} \Phi(t,|x(t)|) d \mu(t)$. The space

$$
L^{\Phi}(\mu)=\left\{x \in L^{0}(\mu): I_{\Phi}(\lambda x)<\infty \text { for some } \lambda>0\right\}
$$

is said to be a Musielak-Orlicz space (see [9-11]). We will also consider the subspace $E^{\Phi}(\mu)$ of $L^{\Phi}(\mu)$ :

$$
E^{\Phi}(\mu)=\left\{x \in L^{0}(\mu): I_{\Phi}(\lambda x)<\infty \text { for any } \lambda>0\right\} .
$$


These spaces are complete with respect to the following Mazur-Orlicz F-norm (see $[9-11])$ :

$$
\|x\|_{\Phi}=\inf \left\{a>0: I_{\Phi}(x / a) \leq a\right\} \quad \forall x \in L^{\Phi}(\mu) .
$$

These spaces are $F$-lattices when considered with the natural partial order.

In the whole paper we will write shortly $L^{\Phi}(\mu)$ and $E^{\Phi}(\mu)$ instead of $\left(L^{\Phi}(\mu),\|\cdot\|_{\Phi}\right)$ and $\left(E^{\Phi}(\mu),\|\cdot\|_{\Phi}\right)$, respectively, assuming that these function spaces are generated by a monotone Musielak-Orlicz function $\Phi$.

Let us present now some useful results. The proof of Lemma 1.8 will be omitted because it is the same as the proof of implications $(i) \Rightarrow(i i) \Rightarrow(i i i)$ of Theorem 5.5 from [2], where, in fact, the continuity of the function $\Phi(t,$. for $\mu$-a.e. $t \in \Omega$ was important.

Lemma 1.8. (cf. [2], Theorem 5.5). If $\Phi \notin \Delta_{2}$, then $D_{\Phi}=\left\{t \in \Omega: b_{\Phi}(t)<\infty\right\}$ is a nonnull set or for any

$$
b_{1} \geq b_{2} \geq \cdots>1, \quad 1<p_{1} \leq p_{2} \leq \cdots, \quad q_{n}>0 \quad(n \in \mathbb{N}),
$$

there exist $\mu$-measurable functions $\left\{x_{n}(t)\right\}_{n=1}^{\infty}$ and mutually disjoint $\left\{F_{n}\right\}_{n=1}^{\infty}$ in $\Sigma$ such that $x_{n}(t)<\infty$ on $F_{n}$ and

$$
\int_{F_{n}} \Phi\left(t, x_{n}(t)\right) d t=q_{n}, \quad \Phi\left(t, b_{k_{n}} x_{n}(t)\right) \geq p_{k_{n}} \Phi\left(t, x_{n}(t)\right) \quad\left(t \in F_{n}, n \in \mathbb{N}\right),
$$

where $\left\{k_{n}\right\}$ is a subsequence of $\mathbb{N}$.

Theorem 1.9. (cf. [10], Theorem 8.13 (a)). If $\mu$ is $\sigma$-finite and atomless and $\Phi$ is a monotone Musielak-Orlicz function such that $a_{\Phi}(t)=0$ and $b_{\Phi}(t)=\infty$ for $\mu$-a.e. $t \in \Omega$, then $E^{\Phi}(\mu)=L^{\Phi}(\mu)$ if and only if $\Phi \in \Delta_{2}$.

Remark 1.10. Theorem 1.9 also holds true without the assumption that a generating monotone Musielak-Orlicz function $\Phi$ is such that $a_{\Phi}(t)=0$ for $\mu$-a.e. $t \in \Omega$.

Lemma 1.11. (see [8], Lemma 1.7.3). Let $\left\{\alpha_{i}\right\}_{i=1}^{\infty}$ be a sequence of positive numbers and $\left\{a_{i}(.)\right\}_{i=1}^{\infty}$ be a sequence of finite, non-negative functions such that $\int_{\Omega} a_{i}(t) d \mu(t) \geq 2^{i} \alpha_{i}, i=1,2, \ldots$. If $\mu$ is non-atomic, then there exists a subsequence $\left\{i_{k}\right\}_{k=1}^{\infty}$ of integers and a collection $\left\{A_{k}\right\}_{k=1}^{\infty}$ of disjoint subsets of $\Omega$ such that

$$
\int_{A_{k}} a_{i_{k}}(t) d \mu(t)=\alpha_{i_{k}} \quad \text { for } k=1,2, \ldots
$$

Remark 1.12. The collection $\left\{A_{k}\right\}_{k=1}^{\infty}$ of disjoint sets from Lemma 1.11 can be found in such a way that $\mu\left(\Omega \backslash \bigcup_{k=1}^{\infty} A_{k}\right)>0$ (see the proof of Lemma 1.7.3 in [8]). 
The results presented in the paper generalize some of the results from papers $[3,5]$, obtained there for Orlicz spaces and their subspaces of order continuous elements endowed with the Mazur-Orlicz F-norm. However, the proofs of the results presented in this paper require deeper techniques then the ones which concern only Orlicz spaces. The application of some methods or ideas of proofs from papers $[2-6,8]$ was useful.

It seems to be interesting to note that the case of Orlicz spaces equipped with the Mazur-Orlicz F-norm already shows that, for instance, the characterization of strict monotonicity and orthogonal strict monotonicity differ (see $[3,5])$, which was not possible in the norm case.

\section{Results}

Let us start with presenting a generalization of Lemma 6.1 from [5].

Lemma 2.1. For every monotone Musielak-Orlicz function $\Phi$, any $x \in L^{\Phi}(\mu)$ $\backslash\{0\}$ and any Mazur-Orlicz F-norm $\|\cdot\|_{\Phi}$, we have:

(1) $I_{\Phi}\left(\frac{x}{\|x\|_{\Phi}}\right) \leq\|x\|_{\Phi}$.

(2) If $I_{\Phi}\left(\lambda \frac{x}{\|x\|_{\Phi}}\right)<\infty$ for some $\lambda>1$, then $I_{\Phi}\left(\frac{x}{\|x\|_{\Phi}}\right)=\|x\|_{\Phi}$.

(3) If $I_{\Phi}\left(\frac{x}{\lambda}\right)=\lambda$ for $\lambda>0$, then $\|x\|_{\Phi}=\lambda$.

Proof. Although the proof of statements (1) and (2) goes in the same way as the proof of Lemma 6.1 from [5] and the proof of statement (3) is the same as that in [3, Lemma 2.16(iii)], we will present them just for the convenience of the reader.

The proof of statement (1). Note that function $f(\lambda)=I_{\Phi}\left(\frac{x}{\lambda}\right)$ is non-increasing on $(0, \infty)$ for any $x \in L^{\Phi}(\mu)$. From the definition of the Mazur-Orlicz $F$-norm $\|\cdot\|_{\Phi}$, we obtain that if $x \in L^{\Phi}(\mu)$, then $I_{\Phi}\left(\frac{x}{\|x\|_{\Phi}+\varepsilon}\right) \leq\|x\|_{\Phi}+\varepsilon$ for any $\varepsilon>0$. Let us take a sequence $\left(\varepsilon_{n}\right)_{n=1}^{\infty}$ of positive numbers such that $\varepsilon_{n} \searrow 0$ as $n \rightarrow \infty$. Then

$$
I_{\Phi}\left(\frac{x}{\|x\|_{\Phi}+\varepsilon_{n}}\right) \leq\|x\|_{\Phi}+\varepsilon_{n}
$$

for any $n \in \mathbb{N}$, whence by the Beppo Levi theorem, we get that $I_{\Phi}\left(\frac{x}{\|x\|_{\Phi}}\right) \leq$ $\|x\|_{\Phi}$ for any $x \in L^{\Phi}(\mu) \backslash\{0\}$.

The proof of statement (2). Assume that $I_{\Phi}\left(\lambda \frac{x}{\|x\|_{\Phi}}\right)<\infty$ for some $\lambda>1$ and for any $x \in L^{\Phi}(\mu)$. For any sequence $\left(\varepsilon_{n}\right)_{n=1}^{\infty}$ in $\left(0,\|x\|_{\Phi}\right)$ such that $\varepsilon_{n} \searrow 0$ as $n \rightarrow \infty$ and $\varepsilon_{1} \in\left(0,\|x\|_{\Phi}\right)$ satisfying inequality the $\frac{1}{\|x\|_{\Phi}-\varepsilon_{1}} \leq \frac{\lambda}{\|x\|_{\Phi}}$, the condition

$$
I_{\Phi}\left(\frac{x}{\|x\|_{\Phi}-\varepsilon_{n}}\right)>\|x\|_{\Phi}-\varepsilon_{n}
$$


holds for any $n \in \mathbb{N}$. Since $\Phi\left(t, \frac{|x(t)|}{\|x\|_{\Phi}-\varepsilon_{n}}\right) \leq \Phi\left(t, \frac{|x(t)|}{\|x\|_{\Phi}-\varepsilon_{1}}\right) \in L^{1}(\mu)$ for $\mu$-a.e. $t \in \Omega$ and $\Phi\left(t, \frac{|x(t)|}{\|x\|_{\Phi}-\varepsilon_{n}}\right) \searrow \Phi\left(t, \frac{|x(t)|}{\|x\|_{\Phi}}\right)$ as $n \rightarrow \infty$ and for $\mu-$ a.e. $t \in \Omega$, by the Lebesgue dominated convergence theorem, we obtain

$$
I_{\Phi}\left(\frac{x}{\|x\|_{\Phi}-\varepsilon_{n}}\right) \searrow I_{\Phi}\left(\frac{x}{\|x\|_{\Phi}}\right)
$$

as $n \rightarrow \infty$, which jointly with inequality (2.1) gives $I_{\Phi}\left(\frac{x}{\|x\|_{\Phi}}\right) \geq\|x\|_{\Phi}$. By virtue of statement (1) of Lemma 2.1, we conclude that $I_{\Phi}\left(\frac{x}{\|x\|_{\Phi}}\right)=\|x\|_{\Phi}$ for any $x \in L^{\Phi}(\mu) \backslash\{0\}$, which ends the proof of the Lemma.

The proof of statement (3). By the definition of the F-norm $\|\cdot\|_{\Phi}$, we have that $\|x\|_{\Phi} \leq \lambda$. Assuming that $\|x\|_{\Phi}<\lambda$, by statement (1) of this lemma, we get

$$
\lambda=I_{\Phi}\left(\frac{x}{\lambda}\right) \leq I_{\Phi}\left(\frac{x}{\|x\|_{\Phi}}\right) \leq\|x\|_{\Phi}<\lambda,
$$

a contradiction.

Corollary 2.2. In the space $L^{\Phi}(\mu)$ the following relationships between the modular $I_{\Phi}$ and the F-norm $\|\cdot\|_{\Phi}$ hold:

(1) $I_{\Phi}(x) \leq\|x\|_{\Phi}$ whenever $x \in B\left(L^{\Phi}(\mu)\right)$.

(2) If $x \in S\left(L^{\Phi}(\mu)\right)$ and $I_{\Phi}(\lambda x)<\infty$ for some $\lambda>1$, then $I_{\Phi}(x)=1$.

(3) The equality $I_{\Phi}\left(\frac{x}{\|x\|_{\Phi}}\right)=\|x\|_{\Phi}$ holds for every $x \in L^{\Phi}(\mu) \backslash\{0\}$ whenever $\Phi \in \Delta_{2}$.

Proof. Statement (1) follows directly from statement (1) of Lemma 2.1 and statements (2) and (3) come from statement (2) of Lemma 2.1 because the assumption that $\Phi \in \Delta_{2}$ gives that $I_{\Phi}(\lambda x)<\infty$ for any $\lambda>0$.

Remark 2.3. Note that the $\Delta_{2}$-condition for $\Phi$ implies that $b_{\Phi}(t)=\infty$ for $\mu$-a.e. $t \in \Omega$, so in statement (3) of Corollary 2.2, the assumption that $b_{\Phi}(t)=$ $\infty$ for $\mu$-a.e. $t \in \Omega$ is satisfied automatically.

Theorem 2.4. If $a_{\Phi}(t)>0$ for $t$ from a measurable set of positive measure, then the space $L^{\Phi}(\mu)$ is not orthogonally strictly monotone.

Proof. Assume that $a_{\Phi}(t)>0$ for $t \in A \subset \Omega$ with $\mu(A)>0$. Passing to a subset, if necessary, and denoting it again by $A$, we can assume that $\mu(A)<\infty$ and $\mu(\Omega \backslash A)>0$. We will consider two cases separately.

Case 1. Let $b_{\Phi}(t)=\infty$ for $\mu$-a.e. $t \in \Omega$. Then, there exists a measurable set $B \subset \Omega \backslash A$ such that $0<\int_{B} \Phi\left(t, a_{\Phi}(t)+1\right) d \mu=b<\infty$. Define

$$
y(t)=\left(a_{\Phi}(t)+1\right) \chi_{B}(t), \quad x(t)=a_{\Phi}(t) \chi_{A}(t)+\left(a_{\Phi}(t)+1\right) \chi_{B}(t) .
$$

Then $I_{\Phi}(y)=I_{\Phi}(x)=b$, so $y, x \in L^{\Phi}(\mu)$. Writing the last equalities equivalently, we get that $I_{\Phi}\left(\frac{b y}{b}\right)=I_{\Phi}\left(\frac{b x}{b}\right)=b$, which by statement (3), Lemma 2.1, 
yields that $\|b y\|_{\Phi}=\|b x\|_{\Phi}=b$. But $0 \leq b y \leq b x$ and $b y \neq b x$, so $L^{\Phi}(\mu)$ is not orthogonally strictly monotone.

Case 2. Let $b_{\Phi}(t)<\infty$ on a measurable set $A$ of positive and finite measure. We will consider two subcases.

Subcase 1. Assume $a_{\Phi}(t)=b_{\Phi}(t)$ for $\mu$-a.e. $t \in A$. Then, taking a set $B \subset A$ such that $0<\mu(B)<\mu(A)<\infty$ and defining

$$
y(t)=b_{\Phi}(t) \chi_{B}(t), \quad x(t)=b_{\Phi}(t) \chi_{A}(t),
$$

we get that $0 \leq y \leq x, y \neq x$ and $I_{\Phi}(y)=I_{\Phi}(x)=0$. Moreover, $I_{\Phi}\left(\frac{x}{\lambda}\right)=$ $I_{\Phi}\left(\frac{y}{\lambda}\right)=\infty$ for any $\lambda \in(0,1)$. Therefore, $\|x\|_{\Phi}=\|y\|_{\Phi}=1$, so $L^{\Phi}(\mu)$ is not orthogonally strictly monotone.

Subcase 2. Assume $a_{\Phi}(t)<b_{\Phi}(t)$ on a measurable set $B$ of positive measure such that $B \subset A$. Define the sets

$$
B_{n}=\left\{t \in B: a_{\Phi}(t)+\frac{1}{n}<b_{\Phi}(t)\right\} .
$$

Then $B_{n} \subseteq B_{n+1}$ and $\bigcup_{n=1}^{\infty} B_{n}=B$ whence there exists $m \in \mathbb{N}$ such that $\mu\left(B_{m}\right)>0$. Denote $\int_{B_{m}} \Phi\left(a_{\Phi}(t)+\frac{1}{m}\right) d \mu=\widetilde{c} \leq \infty$. Then, there exists $C_{m} \subset$ $B_{m}$ of positive measure such that $\int_{C_{m}} \Phi\left(a_{\Phi}(t)+\frac{1}{m}\right) d \mu=: c<\infty$. Let us define

$$
y(t)=\left(a_{\Phi}(t)+\frac{1}{m}\right) \chi_{C_{m}}, \quad x(t)=y(t)+a_{\Phi}(t) \chi_{A \backslash B} .
$$

Then $I_{\Phi}(y)=I_{\Phi}(x)=c$, so $x, y \in L^{\Phi}(\mu)$. Writing the last equalities equivalently in the form $I_{\Phi}\left(\frac{c y}{c}\right)=I_{\Phi}\left(\frac{c x}{c}\right)=c$, by statement (3) of Lemma 2.1, we get that $\|c y\|_{\Phi}=\|c x\|_{\Phi}=c$. However, $0 \leq c y \leq c x$ and $c y \neq c x$, so again $L^{\Phi}(\mu)$ is not orthogonally strictly monotone.

Remark 2.5. If we assume additionally in Theorem 2.4 that $b_{\Phi}(t)=\infty$ for $\mu$-a.e. $t \in \Omega$, then we can present the following simpler proof of this theorem.

If $a_{\Phi}(t)>0$ for $t$ belonging to a measurable set $B$ of positive measure, then we can find (coming to a subset $A$ of the set $B$, if necessary) $a, b \in \mathbb{R}_{+}$such that $0<a<b \leq a_{\Phi}(t)$ and $\Phi(t, a)=\Phi(t, b)$ for all $t \in A \subset B$. Indeed, let us define sets $B_{n}:=\left\{t \in B: \frac{1}{n}<a_{\Phi}(t)\right\}$. Then $\bigcup_{n=1}^{\infty} B_{n}=B$, so there exists $l \in \mathbb{N}$ such that $\mu\left(B_{l}\right)>0$. Therefore, $0<\frac{1}{l+1}<\frac{1}{l}<a_{\Phi}(t)$ for all $t \in B_{l}$. Setting $a=\frac{1}{l+1}, b=\frac{1}{l}$ and $A=B_{l}$, we have shown our thesis.

Next, there exists an increasing sequence $\left(A_{n}\right)_{n=1}^{\infty}$ of measureable sets such that $\mu\left(A_{n}\right)<\infty, \mu\left(\Omega \backslash \bigcup_{n=1}^{\infty} A_{n}\right)=0$ and $\sup _{t \in A_{n}} \Phi(t, u)<\infty$ for all $u \in \mathbb{R}_{+}$ and $n \in \mathbb{N}$ (see [6]), which gives that $\chi_{A_{n}} \in E^{\Phi}(\mu)$ for all $n \in \mathbb{N}$. There exists $m \in \mathbb{N}$ such that $\mu\left(A \cap A_{m}\right)>0$, whence $\Phi(t, \lambda) \chi_{A \cap A_{m}} \in L^{1}(\mu)$ for any $\lambda>0$. 
Let $F \subset A \cap A_{m}$ be a set of positive (and finite) measure. Divide this set into two disjoint measurable subsets $C$ and $D$ of positive measures such that $C \cup D=F$. Let us define

$$
y(t)=\left(a+a_{\Phi}(t)\right) \chi_{C}(t) \text { and } x(t)=\left(a+a_{\Phi}(t)\right) \chi_{C}(t)+a_{\Phi}(t) \chi_{D}(t) .
$$

Denote $w=I_{\Phi}\left(\left[a+a_{\Phi}(t)\right] \chi_{C}\right)$. Then $0 \leq y \leq x, y \neq x$ and

$$
I_{\Phi}(y)=I_{\Phi}(x)=w<\infty,
$$

so $y, x \in L^{\Phi}(\mu)$. Writing equations (2.2) equivalently, we obtain $I_{\Phi}\left(\frac{w y}{w}\right)=$ $I_{\Phi}\left(\frac{w x}{w}\right)=w$, which by statement (3) of Lemma 2.1, yields that $\|w y\|_{\Phi}=$ $\|w x\|_{\Phi}=w$. But $0 \leq w y \leq w x$ and $w y \neq w x$, so $L^{\Phi}(\mu)$ is not orthogonally strictly monotone.

Theorem 2.6. If $b_{\Phi}(t)<\infty$ for $t$ from a measurable set of positive measure, then the space $L^{\Phi}(\mu)$ is not orthogonally strictly monotone.

Proof. Assume that $b_{\Phi}(t)<\infty$ on a set of positive measure. Although the proof goes similarly to the proof of Theorem 1.4 from [4], we will present it just for the convenience of the reader. Denote

$$
A_{0}=\left\{t \in \Omega: b_{\Phi}(t)<\infty\right\}
$$

and let $\left(u_{i}\right)_{i=1}^{\infty}$ denote the sequence of positive rational numbers. Then

$$
A_{0}=\bigcup_{i \in \mathbb{N}} \bigcap_{n \in \mathbb{N}}\left\{t \in \Omega: \Phi\left(t, u_{i}\right) \geq n\right\} \in \Sigma .
$$

We also know that $b_{\Phi}($.$) is a \Sigma$-measurable function. Since $b_{\Phi}(t)<\infty$ for $t$ from a set of positive measure, $\mu\left(A_{0}\right)>0$. Let $A \subset A_{0}$ be a measurable subset of the set $A_{0}$ such that $\mu(A) \in(0, \infty)$ and let $u(t)=b_{\Phi}(t) \chi_{A}(t)$. Let $\left\{A_{k}\right\}_{k=1}^{\infty}$ be a sequence of pairwise disjoint and measurable subsets of $A$ of positive measure and let $\left\{\alpha_{k}\right\}_{k=1}^{\infty}$ be a sequence of real numbers such that $0<\alpha_{k}<1$ for any $k \in \mathbb{N}$ and $\alpha_{k} \uparrow 1$ as $k \rightarrow \infty$. Define

$$
A_{k}^{n}=\left\{t \in A_{k}: \Phi\left(t, \alpha_{k} u(t)\right) \leq n\right\}, \quad k, n \in \mathbb{N} .
$$

Then $A_{k}^{n} \subset A_{k}^{m}$ for $n \leq m$. Thus $0<\lim _{n \rightarrow \infty} \mu\left(A_{k}^{n}\right)=\mu\left(A_{k}\right)<\infty$ for all $k \in \mathbb{N}$. Hence, for every $k \in \mathbb{N}$ we can find an integer $n(k)$ such that $\mu\left(A_{k}^{n(k)}\right)>0$. Further, for every $k \in \mathbb{N}$ there exists a set $B_{k} \in \Sigma, B_{k} \subset A_{k}^{n(k)}$ such that $\mu\left(B_{k}\right)>0$ and $\int_{B_{k}} \Phi\left(t, \alpha_{k} u(t)\right) d \mu \leq 2^{-k}$. Set $x_{k}(t)=\alpha_{k} u(t) \chi_{B_{k}}(t)$ and define

$$
x(t)=\sum_{k=1}^{\infty} x_{k}(t), \quad y(t)=\sum_{k=2}^{\infty} x_{k}(t) .
$$

Then $0 \leq y \leq x, y \neq x$ and

$$
I_{\Phi}(x)=\sum_{k=1}^{\infty} \int_{B_{k}} \Phi\left(t, \alpha_{k} u(t)\right) d \mu \leq \sum_{k=1}^{\infty} \frac{1}{2^{k}}=1,
$$


whence $\|y\|_{\Phi} \leq\|x\|_{\Phi} \leq 1$, so $x, y \in L^{\Phi}(\mu)$. Moreover, for any $\lambda \in(0,1)$ we can find $k_{0} \in \mathbb{N}, k_{0} \geq 2$ such that $\lambda<\alpha_{k}$ for any $k \geq k_{0}$. Hence,

$$
I_{\Phi}\left(\frac{y}{\lambda}\right) \geq \sum_{k=k_{0}}^{\infty} \int_{B_{k}} \Phi\left(t, \frac{\alpha_{k} u(t)}{\lambda}\right) d \mu=\infty,
$$

so $\|y\|_{\Phi} \geq \lambda$. By the arbitrariness of $\lambda \in(0,1)$, we obtain that $\|y\|_{\Phi} \geq 1$. Hence and by the fact that $\|x\|_{\Phi} \leq 1$, we conclude that $\|x\|_{\Phi}=\|y\|_{\Phi}=1$, so $L^{\Phi}(\mu)$ is not strictly monotone.

Remark 2.7. Another, simpler proof of Theorem 2.6 is possible and it looks as follow.

Assume that the set $A=\left\{t \in \Omega: b_{\Phi}(t)<\infty\right\}$ has a positive measure. Let $\left\{A_{n}\right\}_{n=1}^{\infty}$ be a sequence of pairwise disjoint sets in $\Sigma \cap A$. Let us choose for any $n \in \mathbb{N}$ a measurable subset $B_{n}$ of $A_{n}$ such that $\mu\left(B_{n}\right)>0$ and $I_{\Phi}\left(\left(1-\frac{1}{n+1}\right) b_{\Phi} \chi_{B_{n}}\right) \leq 2^{-n}$. Define $x(t)=\sum_{n=1}^{\infty}\left(1-\frac{1}{n+1}\right) b_{\Phi}(t) \chi_{B_{n}}(t), \quad y(t)=\sum_{n=2}^{\infty}\left(1-\frac{1}{n+1}\right) b_{\Phi}(t) \chi_{B_{n}}(t)$. Then $0 \leq y \leq x, y \neq x$ and $I_{\Phi}(y) \leq I_{\Phi}(x) \leq \sum_{n=1}^{\infty} 2^{-n}=1$, whence $\|y\|_{\Phi} \leq$ $\|x\|_{\Phi} \leq 1$. Taking any $\lambda \in(0,1)$ one can find $n_{\lambda} \in \mathbb{N}$ such that $\frac{1-\frac{1}{n+1}}{\lambda}>1$ for any $n \geq n_{\lambda}$, whence $I_{\Phi}\left(\frac{x}{\lambda}\right) \geq I_{\Phi}\left(\frac{y}{\lambda}\right) \geq \sum_{n=n_{\lambda}}^{\infty} I_{\Phi}\left(\frac{1-\frac{1}{n+1}}{\lambda} b_{\Phi} \chi_{B_{n}}\right)=\infty$. Consequently, $\|x\|_{\Phi} \geq\|y\|_{\Phi} \geq \lambda$. By the arbitrariness of $\lambda \in(0,1)$, we get that $\|y\|_{\Phi} \geq 1$, whence $\|y\|_{\Phi}=\|x\|_{\Phi}=1$, so $L^{\Phi}(\mu)$ is not orthogonally strictly monotone.

Theorem 2.8. The space $L^{\Phi}(\mu)$ is strictly monotone if and only if $\Phi(t,$.$) is$ strictly increasing for $\mu-$ a.e. $t \in \Omega$ and $\Phi \in \Delta_{2}$.

Proof. Sufficiency. Assume that $\Phi(t,$.$) is increasing for \mu$-a.e. $t \in \Omega$ and $\Phi \in \Delta_{2}$. Then, taking any $0 \leq y \leq x \in L^{\Phi}(\mu), y \neq x$ and assuming that $\|x\|_{\Phi}=\|y\|_{\Phi}$, by statement (2) of Lemma 2.1, we obtain

$$
\|y\|_{\Phi}=I_{\Phi}\left(\frac{y}{\|y\|_{\Phi}}\right)<I_{\Phi}\left(\frac{x}{\|y\|_{\Phi}}\right)=I_{\Phi}\left(\frac{x}{\|x\|_{\Phi}}\right)=\|x\|_{\Phi},
$$

a contradiction. Hence $\|y\|_{\Phi}<\|x\|_{\Phi}$, so $L^{\Phi}(\mu)$ is strictly monotone.

Necessity. By virtue of Theorem 2.6 we can assume that $b_{\Phi}(t)=\infty$ for $\mu$-a.e. $t \in \Omega$ because otherwise $L^{\Phi}(\mu)$ is not even orthogonally strictly monotone. Under this assumption, first we will show that if $\Phi \notin \Delta_{2}$, then $L^{\Phi}(\mu)$ is not strictly monotone. In order to do so, assume that $b_{\Phi}(t)=\infty$ for $\mu$-a.e. $t \in \Omega$ but $\Phi \notin \Delta_{2}$. Applying Lemma 1.8 with $b_{n}=1+\frac{1}{n}, p_{n}=2^{n}$ and $q_{n}=\frac{1}{2^{n}}$, 
where $n \in \mathbb{N}$, we can find a sequence $\left\{\widetilde{x}_{n}(t)\right\}_{n=1}^{\infty}$ of $\Sigma$-measurable functions and mutually disjoint sets $\left\{F_{n}\right\}_{n=1}^{\infty}$ in $\Sigma$ such that $\widetilde{x}_{n}(t)<\infty$ on the set $F_{n}$ and we have

$$
\int_{F_{n}} \Phi\left(t, \widetilde{x}_{n}(t)\right) d t=\frac{1}{2^{n}}, \quad \Phi\left(t, b_{k_{n}} \widetilde{x}_{n}(t)\right) \geq p_{k_{n}} \Phi\left(t, \widetilde{x}_{n}(t)\right) \quad\left(t \in F_{n}, n \in \mathbb{N}\right),
$$

where $\left\{k_{n}\right\}$ is a strictly increasing subsequence of $\mathbb{N}$. Let us define

$$
y(t)=\sum_{n=2}^{\infty} \widetilde{x}_{n}(t) \chi_{F_{n}}(t) \text { and } x(t)=\sum_{n=1}^{\infty} \widetilde{x}_{n}(t) \chi_{F_{n}}(t) .
$$

Then $0 \leq y \leq x, y \neq x$ and $I_{\Phi}(x)=\sum_{n=1}^{\infty} \Phi\left(t, \widetilde{x}_{n}(t)\right) \mu\left(F_{n}\right)=\sum_{n=1}^{\infty} \frac{1}{2^{n}}=1$, whence $y, x \in L^{\Phi}(\mu)$ and, by statement (3) of Lemma 2.1, we get that $\|x\|_{\Phi}=1$. Moreover, $I_{\Phi}(y)<1$ and for any $\lambda \in(0,1)$ there exists $m \in \mathbb{N}, m \geq 2$ such that $\frac{1}{\lambda}>1+\frac{1}{k_{n}}$ for any $n \geq m$, whence

$$
\begin{aligned}
I_{\Phi}\left(\frac{y}{\lambda}\right) & \geq \sum_{n=m}^{\infty} \Phi\left(t, \frac{\widetilde{x}_{n}(t)}{\lambda}\right) \mu\left(F_{n}\right) \geq \sum_{n=m}^{\infty} \Phi\left(t,\left(1+\frac{1}{k_{n}}\right) \widetilde{x}_{n}(t)\right) \mu\left(F_{n}\right) \\
& \geq \sum_{n=m}^{\infty} 2^{n} \Phi\left(t, \widetilde{x}_{n}(t)\right) \mu\left(F_{n}\right)=\sum_{n=m}^{\infty} 1=\infty
\end{aligned}
$$

which gives that $\|y\|_{\Phi} \geq \lambda$ and, consequently, $\|y\|_{\Phi} \geq 1$ by the arbitrariness of $\lambda \in(0,1)$. Therefore $\|y\|_{\Phi}=\|x\|_{\Phi}=1$, which means that $L^{\Phi}(\mu)$ is not strictly monotone.

Now, we will show the necessity of the assumption that $\Phi(t,$.$) is strictly$ increasing on $\mathbb{R}_{+}$for $\mu$-a.e. $t \in \Omega$. Assume that $b_{\Phi}(t)=\infty$ for $\mu$-a.e. $t \in \Omega$ and that $\mu\left(\Omega_{n s i}\right)>0$, where $\Omega_{n s i}=\left\{t \in \Omega: \Phi(t,\right.$.$\left.) is not strictly increasing on \mathbb{R}_{+}\right\}$. Then, passing to a subset if necessary, there exist a set $A$ of positive and finite measure and $a, b \in \mathbb{R}_{+}$such that $0<a<b<\infty$ and $\Phi(t, a)=\Phi(t, b)$ for all $t \in A$. Indeed, since the set of positive rational numbers $\mathbb{Q}_{+}$is countable, we can write $\mathbb{Q}_{+}=\left\{r_{n}\right\}_{n=1}^{\infty}$, where $r_{n} \neq r_{m}$ for any $m, n \in \mathbb{N}$ such that $m \neq n$. For $r_{m}, r_{n} \in \mathbb{Q}_{+}$such that $r_{m}<r_{n}$, let us define the sets

$$
A_{m, n}=\left\{t \in \Omega_{n s i}: \Phi\left(t, r_{m}\right)=\Phi\left(t, r_{n}\right)\right\} \text {. }
$$

Since $\bigcup_{\substack{m, n=1 \\ m \neq n}}^{\infty} A_{m, n}=\Omega_{n s i}$, there exist $m_{0}, n_{0} \in \mathbb{N}$ such that $m_{0} \neq n_{0}, \mu\left(A_{m_{0}, n_{0}} \cap\right.$ $\left.\Omega_{n s i}\right)>0$ and we have that $\Phi\left(t, r_{m_{0}}\right)=\Phi\left(t, r_{n_{0}}\right)$ for any $t \in A_{m_{0}, n_{0}}$, so it is enough to set $a=r_{m_{0}}, b=r_{n_{0}}$ and $A=A_{m_{0}, n_{0}}$.

Next, there exists an increasing sequence $\left(A_{n}\right)_{n=1}^{\infty}$ of sets such that $\mu\left(A_{n}\right)<$ $\infty, \mu\left(\Omega \backslash \bigcup_{n=1}^{\infty} A_{n}\right)=0$ and $\sup _{t \in A_{n}} \Phi(t, u)<\infty$ for all $u \in \mathbb{R}_{+}$and $n \in \mathbb{N}$ (see [6]), which gives that $\chi_{A_{n}} \in E^{\Phi}(\mu)$ for all $n \in \mathbb{N}$. Then, there exists $m \in \mathbb{N}$ 
such that $\mu\left(A \cap A_{m}\right)>0$, whence $\Phi(t, \lambda) \chi_{A \cap A_{m}} \in L^{1}(\mu)$ for any $\lambda>0$. We will consider two cases separately.

Case 1. Let $\Phi(t, a)>0$ for any $t \in A$. Define $y=a \chi_{A \cap A_{m}}$ and $x=b \chi_{A \cap A_{m}}$ and let us denote $d=I_{\Phi}\left(a \chi_{A \cap A_{m}}\right)$. Then $0 \leq y \leq x, y \neq x$ and $I_{\Phi}(y)=$ $\int_{A \cap A_{m}} \Phi(t, a) d \mu(t)=\int_{A \cap A_{m}} \Phi(t, b) d \mu(t)=I_{\Phi}(x)$, so $I_{\Phi}(y)=I_{\Phi}(x)=d<\infty$, whence $y, x \in L^{\Phi}(\mu)$. Writing the last equalities equivalently, we obtain that $I_{\Phi}\left(\frac{d y}{d}\right)=I_{\Phi}\left(\frac{d x}{d}\right)=d$, whence by statement (3) of Lemma 2.1, we get that $\|d y\|_{\Phi}=\|d x\|_{\Phi}=d$. By virtue of the facts that $0 \leq d y \leq d x$ and $d y \neq d x$, we conclude that $L^{\Phi}(\mu)$ is not strictly monotone.

Case 2. Let us assume that $\Phi(t, a)=0$ for any $t \in A$. Then $a_{\Phi}(t)>0$ for $t \in A$. By virtue of Theorem 2.4, in this case $L^{\Phi}(\mu)$ is not even orthogonally strictly monotone.

Let us present now a useful lemma.

Lemma 2.9. If $(\Omega, \Sigma, \mu)$ is a non-atomic, complete and $\sigma$-finite measure space and $\Phi$ is a monotone Musielak-Orlicz function, then the space $E^{\Phi}(\mu)$ is nontrivial if and only if the set $\Omega_{o c}$ has positive measure. Moreover, in this case $\operatorname{supp} E^{\Phi}(\mu)=\Omega_{o c}$, that is, there exists a function $x \in E^{\Phi}(\mu)$ such that $x(t)>0$ for any $t \in \Omega_{o c}$ as well as if $x \in L^{0}(\mu)$ and $\mu\left[\left(\Omega \backslash \Omega_{o c}\right) \cap \operatorname{supp} x\right]>0$ then $x \notin E^{\Phi}(\mu)$.

Proof. Sufficiency. Assume that $\mu\left(\Omega_{o c}\right)>0$. Then (see [6]), there exists a sequence of increasing sets $\left\{A_{n}\right\}_{n=1}^{\infty}$ in $\Omega_{o c} \cap \Sigma$ such that $\chi_{A_{n}} \in E^{\Phi}(\mu), 0<$ $\mu\left(A_{n}\right)<\infty$ and $\bigcup_{n=1}^{\infty} A_{n}=\Omega_{o c}$. This shows that $E^{\Phi}(\mu) \neq\{0\}$.

Necessity. Assume that $x \in L^{0}(\mu)$ and $\mu\left[\left(\Omega \backslash \Omega_{o c}\right) \cap \operatorname{supp} x\right]>0$. Let us denote $A_{x}=\left(\Omega \backslash \Omega_{o c}\right) \cap \operatorname{supp} x$. Then $b_{\Phi}(t)<\infty$ for any $t \in A_{x}$. Let us define

$$
A_{x, n}=\left\{t \in A_{x}:|x(t)|>\frac{1}{n}\right\} \quad(\forall n \in \mathbb{N}) .
$$

Then, $A_{x, n} \subset A_{x, n+1}$ for any $n \in \mathbb{N}$ and $\bigcup_{n=1}^{\infty} A_{x, n}=A_{x}$. Therefore, there exists $m \in \mathbb{N}$ such that $\mu\left(A_{x, m}\right)>0$ and $b_{\Phi}(t)<\infty$ for all $t \in A_{x, m}$. Now, let us define the set

$$
B_{x, n}=\left\{t \in A_{x, m}: b_{\Phi}(t) \leq n\right\}
$$

for any $n \in \mathbb{N}$. It is easy to see that $B_{x, n} \subset B_{x, n+1}$ for any $n \in \mathbb{N}$ and $\bigcup_{n=1}^{\infty} B_{x, n}=$ $A_{x, m}$. Consequently, there exists $k \in \mathbb{N}$ such that $\mu\left(B_{x, k}\right)>0$. Taking $\lambda>0$ so big that $\frac{\lambda}{m}>2 k$, we get $I_{\Phi}(\lambda x) \geq I_{\Phi}\left(\lambda x \chi_{B_{x, k}}\right) \geq I_{\Phi}\left(2 k \chi_{B_{x, k}}\right)=\infty$, by the fact that $\Phi(t, 2 k)=\infty$ for any $t \in B_{x, k}$, which means that $x \notin E^{\Phi}(\mu)$.

Corollary 2.10. The space $E^{\Phi}(\mu)$ is strictly monotone if and only if $\mu\left(\Omega_{o c}\right)=0$ or if $\mu\left(\Omega_{o c}\right)>0$, then $\mu\left(\Omega_{o c} \cap \Omega_{n s i}\right)=0$. 
Proof. Sufficiency. If $\mu\left(\Omega_{o c}\right)=0$, then $E^{\Phi}(\mu)=\{0\}$, whence $E^{\Phi}(\mu)$ is strictly monotone. Assume that $\mu\left(\Omega_{o c}\right)>0$. Then $\mu\left(\Omega_{o c} \cap \Omega_{n s i}\right)=0$. Consequently, $\mu\left(\Omega_{o c} \cap \Omega_{s i}\right)=\mu\left(\Omega_{o c}\right)>0$, where $\Omega_{s i}=\{t \in \Omega: \Phi(t,$.$) is strictly increasing on$ $\left.\mathbb{R}_{+}\right\}$. By virtue of Lemma $2.9, E^{\Phi}(\mu) \neq\{0\}$ and supp $E^{\Phi}(\mu)=\Omega_{o c}$. Therefore, we can (and we will) further consider below the space $E^{\Phi}(\mu)$ on the measure space $\left(\Omega_{o c},\left.\Sigma\right|_{\Omega_{o c}},\left.\mu\right|_{\Omega_{o c}}\right)$ only. Note that $a_{\Phi}(t)=0$ for all $t \in \Omega_{o c}$ (because $\Phi(t,$.$) is strictly increasing on \mathbb{R}_{+}$for $\mu$-a.e. $\left.t \in \Omega_{o c}\right)$. Next, the proof goes in a similar way as the proof of sufficiency for Theorem 2.8 .

Necessity. Assume that $E^{\Phi}(\mu)$ is strictly monotone but $\mu\left(\Omega_{o c}\right)>0$ and $\mu\left(\Omega_{o c} \cap\right.$ $\left.\Omega_{n s i}\right)>0$. Then $\operatorname{supp} E^{\Phi}(\mu)=\Omega_{o c}$, so $b_{\Phi}(t)=\infty$ for all $t \in \Omega_{o c}$. Since the proof goes in a similar way as the proof of the necessity of the fact that $\Phi(t,$. must be strictly increasing on $\mathbb{R}_{+}$for $\mu$-a.e. $t \in \Omega$ in Theorem 2.8, it is omitted (in the case when $a_{\Phi}(t)>0$ on a set of positive measure we can proceed similarly as in the proof of Remark 2.5).

Let us present now Lemmas 2.11 and 2.12 which will be useful in the proof of Theorem 2.13 .

Lemma 2.11. Let $\Phi(t,$.$) be a monotone Musielak-Orlicz function on \mathbb{R}_{+}$for $\mu$-a.e. $t \in \Omega$. Then, for any $x \in E^{\Phi}(\mu)$ the function $f_{x}(\lambda)=I_{\Phi}(\lambda x)$ is continuous on the interval $(0, \infty)$ and right continuous at 0 .

Since the easy proof is similar to the proof of Lemma 4.1(i) from [3], it is omitted.

Lemma 2.12. For any monotone Musielak-Orlicz function $\Phi$, any measure space $(\Omega, \Sigma, \mu)$ and any sequence $\left(x_{n}\right)_{n=1}^{\infty}$ in $L^{\Phi}(\mu)$, modular convergence and $F$ normed convergence are equivalent, i.e. $\left\|x_{n}\right\|_{\Phi} \rightarrow 0$ if and only if $I_{\Phi}\left(\lambda x_{n}\right) \rightarrow 0$ for any $\lambda>0$, as $n \rightarrow \infty$.

Since an easy proof is similar to the proof of Lemma 6.4, p. 2004, from [5], it is omitted.

Theorem 2.13. The following conditions are equivalent:

(a) $E^{\Phi}(\mu)$ is lower locally uniformly monotone,

(b) $E^{\Phi}(\mu)$ is strictly monotone,

(c) $\mu\left(\Omega_{o c}\right)=0$ or if $\mu\left(\Omega_{o c}\right)>0$, then $\mu\left(\Omega_{o c} \cap \Omega_{n s i}\right)=0$.

Proof. By virtue of Corollary 2.10, statements (b) and (c) are equivalent. Moreover, statement (a) implies statement (b). So, we need only to prove that statement (b) implies statement (a).

Note that $E^{\Phi}(\mu)$ is lower locally uniformly monotone if $E^{\Phi}(\mu)=\{0\}$. Assume that $0 \leq x_{n} \leq x \in E_{+}^{\Phi}(\mu) \backslash\{0\}$ for any $n \in \mathbb{N}$ and $\left\|x_{n}\right\|_{\Phi} \rightarrow\|x\|_{\Phi}$ as $n \rightarrow \infty$. We need to prove that $\left\|x_{n}-x\right\|_{\Phi} \rightarrow 0$ as $n \rightarrow \infty$. By virtue of Lemma 2.9 , we know that $\mu\left(\Omega_{o c}\right)>0$ and $\operatorname{supp} E^{\Phi}(\mu)=\Omega_{o c}$. By Lemma 2.1, we get 
that $I_{\Phi}\left(\frac{x_{n}}{\left\|x_{n}\right\|_{\Phi}}\right)=\left\|x_{n}\right\|_{\Phi}$ for any $n \in \mathbb{N}$ and $I_{\Phi}\left(\frac{x}{\|x\|_{\Phi}}\right)=\|x\|_{\Phi}$, whence, by the assumption that $\left\|x_{n}\right\|_{\Phi} \rightarrow\|x\|_{\Phi}$ as $n \rightarrow \infty$, we obtain

$$
I_{\Phi}\left(\frac{x_{n}}{\left\|x_{n}\right\|_{\Phi}}\right) \rightarrow I_{\Phi}\left(\frac{x}{\|x\|_{\Phi}}\right)
$$

as $n \rightarrow \infty$. Next, by Lemma 2.11, $I_{\Phi}\left(\frac{x}{\left\|x_{n}\right\|_{\Phi}}\right) \rightarrow I_{\Phi}\left(\frac{x}{\|x\|_{\Phi}}\right)$ as $n \rightarrow \infty$, which together with condition (2.3) gives

$$
I_{\Phi}\left(\frac{x}{\left\|x_{n}\right\|_{\Phi}}\right)-I_{\Phi}\left(\frac{x_{n}}{\left\|x_{n}\right\|_{\Phi}}\right) \rightarrow 0
$$

as $n \rightarrow \infty$. Since $\Phi\left(t, \frac{x_{n}(t)}{\left\|x_{n}\right\|_{\Phi}}\right) \leq \Phi\left(t, \frac{x(t)}{\left\|x_{n}\right\|_{\Phi}}\right)$ for all $t \in \Omega_{o c}$, condition (2.4) means that

$$
\left\|\Phi\left(t, \frac{x(t)}{\left\|x_{n}\right\|_{\Phi}}\right)-\Phi\left(t, \frac{x_{n}(t)}{\left\|x_{n}\right\|_{\Phi}}\right)\right\|_{L^{1}\left(\Omega_{o c}\right)}=I_{\Phi}\left(\frac{x}{\left\|x_{n}\right\|_{\Phi}}\right)-I_{\Phi}\left(\frac{x_{n}}{\left\|x_{n}\right\|_{\Phi}}\right) \rightarrow 0
$$

as $n \rightarrow \infty$. Consequently, the sequence $\left\{\Phi\left(t, \frac{x(t)}{\left\|x_{n}\right\|_{\Phi}}\right)-\Phi\left(t, \frac{x_{n}(t)}{\left\|x_{n}\right\|_{\Phi}}\right)\right\}_{n=1}^{\infty}$ converges to zero in measure on $\Omega_{o c}$. By the $\sigma$-finiteness of the measure $\mu$, there exists a strictly increasing sequence $\left\{n_{k}\right\}_{k=1}^{\infty}$ in $\mathbb{N}$ such that the sequence $\left\{\Phi\left(t, \frac{x(t)}{\left\|x_{n_{k}}\right\|_{\Phi}}\right)-\Phi\left(t, \frac{x_{n_{k}}(t)}{\left\|x_{n_{k}}\right\|_{\Phi}}\right)\right\}_{k=1}^{\infty}$ converges to zero $\mu$-a.e. in $\Omega_{o c}$. Hence, the assumption that $\Phi(t,$.$) is strictly increasing on \mathbb{R}_{+}$and the continuity of $\Phi(t,$.$) on \mathbb{R}_{+}$for all $t \in \Omega_{o c}$, imply that $\left\{\frac{x}{\left\|x_{n_{k}}\right\|_{\Phi}}-\frac{x_{n_{k}}}{\left\|x_{n_{k}}\right\|_{\Phi}}\right\}_{k=1}^{\infty}$ converges to zero $\mu$-a.e. in $\Omega_{o c}$. This implies that $\left\{x-x_{n_{k}}\right\}_{k=1}^{\infty}$ converges to zero $\mu$-a.e. in $\Omega_{o c}$. Taking arbitrary $\lambda>0$, we get

$$
\Phi\left(t, \lambda\left(x(t)-x_{n_{k}}(t)\right)\right) \rightarrow 0
$$

as $k \rightarrow \infty$ for all $t \in \Omega_{o c}$. Obviously,

$$
\Phi\left(t, \lambda\left[x(t)-x_{n_{k}}(t)\right]\right) \leq \Phi(t, \lambda x(t)) \in L^{1}\left(\Omega_{o c}\right),
$$

for all $t \in \Omega_{o c}$ because $x \in E_{+}^{\Phi}(\mu)$. Conditions (2.6), (2.7) and the Lebesgue dominated convergence theorem yield that $I_{\Phi}\left(\lambda\left(x-x_{n_{k}}\right)\right) \rightarrow 0$ as $k \rightarrow \infty$ for any $\lambda>0$. Consequently, by Lemma 2.12, we conclude that $\left\|x-x_{n_{k}}\right\|_{\Phi} \rightarrow 0$ as $k \rightarrow \infty$. Finally, by the double extract subsequence theorem, the proof is completed.

Corollary 2.14. The following conditions are equivalent:

(a) $L^{\Phi}(\mu)$ is lower locally uniformly monotone,

(b) $L^{\Phi}(\mu)$ is strictly monotone,

(c) $\Phi(t,$.$) is strictly increasing on \mathbb{R}_{+}$for $\mu$-a.e. $t \in \Omega$ and $\Phi \in \Delta_{2}$.

Proof. By virtue of Theorem 2.8, statements (b) and (c) are equivalent. Moreover, it is obvious that statement (a) implies statement (b). Assume that $L^{\Phi}(\mu)$ 
is strictly monotone. Then, by Theorem 2.8, $\Phi$ satisfies condition (c). By Theorem 1.9, we obtain that $L^{\Phi}(\mu)=E^{\Phi}(\mu)$ and it follows from condition (c) that $\mu\left(\Omega \backslash \Omega_{o c}\right)=0$. By virtue of Theorem 2.13 the proof is finished.

Theorem 2.15. The following conditions are equivalent:

(a) $E^{\Phi}(\mu)$ is orthogonally lower locally uniformly monotone,

(b) $E^{\Phi}(\mu)$ is orthogonally strictly monotone,

(c) $\mu\left(\Omega_{o c}\right)=0$ or if $\mu\left(\Omega_{o c}\right)>0$, then $\mu\left(\Omega_{o c} \cap \operatorname{supp} a_{\Phi}\right)=0$.

Proof. The fact that (a) implies (b) is obvious. We will show that (b) implies (c). Assume that statement (b) holds but condition (c) is not satisfied, that is, $\mu\left(\Omega_{o c}\right)>0$ and $\mu\left(\Omega_{o c} \cap \operatorname{supp} a_{\Phi}\right)>0$. We will show that this implies that $E^{\Phi}(\mu)$ is not orthogonally strictly monotone. Take $x \in E_{+}^{\Phi}(\mu) \backslash\{0\}$ with $\mu\left(\Omega_{o c} \backslash \operatorname{supp} x\right)>0$ and a measurable set $A \subset \operatorname{supp} x$ such that $\mu(A)>0$ and $\mu\left(\Omega_{o c} \backslash A\right)>0$. Let us define

$$
y(t)=x(t)+a_{\Phi}(t)\|x\|_{\Phi} \chi_{\left(\Omega_{o c} \backslash \operatorname{supp} x\right) \cap \operatorname{supp} a_{\Phi}}(t) .
$$

Note that $\mu\left[\left(\Omega_{o c} \backslash \operatorname{supp} x\right) \cap \operatorname{supp} a_{\Phi}\right]=\mu\left(\left\{t \in \Omega_{o c}: a_{\Phi}(t)>0=|x(t)|\right\}\right)>0$. Therefore, $0 \leq x \leq y$ and $x \neq y$, so $\|x\|_{\Phi} \leq\|y\|_{\Phi}$. Moreover,

$$
\|y\|_{\Phi}=I_{\Phi}\left(\frac{y}{\|y\|_{\Phi}}\right) \leq I_{\Phi}\left(\frac{y}{\|x\|_{\Phi}}\right)=I_{\Phi}\left(\frac{x}{\|x\|_{\Phi}}\right)=\|x\|_{\Phi},
$$

so $\|y\|_{\Phi} \leq\|x\|_{\Phi}$. Consequently, we have obtained that $\|x\|_{\Phi}=\|y\|_{\Phi}$ and $E^{\Phi}(\mu)$ is not orthogonally strictly monotone.

We will prove now that (c) implies (a). Assume that condition (c) holds. If $\mu\left(\Omega_{o c}\right)=0$, then $E^{\Phi}(\mu)=\{0\}$, so statement (a) holds. If $\mu\left(\Omega_{o c}\right)>0$, then, by the condition $\mu\left(\Omega_{o c} \cap \operatorname{supp} a_{\Phi}\right)=0$, we get that $a_{\Phi}(t)=0$ for $\mu$-a.e. $t \in \Omega_{o c}$. Therefore, without loss of generality, we can consider further only $t \in \Omega_{o c}^{0}:=\left\{t \in \Omega_{o c}: a_{\Phi}(t)=0\right\}$ instead of $t \in \Omega_{o c}$ (sets $\Omega_{o c}$ and $\Omega_{o c}^{0}$ differ only on a measure zero set). Assume that $x \in E_{+}^{\Phi}(\mu) \backslash\{0\}$ and $\left(A_{n}\right)_{n=1}^{\infty}$ is a sequence in $\Sigma \cap \Omega_{o c}^{0}$ such that $\left\|x \chi_{A_{n}}\right\|_{\Phi} \rightarrow\|x\|_{\Phi}$ as $n \rightarrow \infty$. We will show that $\left\|x-x \chi_{A_{n}}\right\|_{\Phi}=\left\|x \chi_{\Omega_{o c}^{0} \backslash A_{n}}\right\|_{\Phi} \rightarrow 0$ as $n \rightarrow \infty$. By statement (2), Lemma 2.1, we get

$$
I_{\Phi}\left(\frac{x \chi_{A_{n}}}{\left\|x \chi_{A_{n}}\right\|_{\Phi}}\right) \rightarrow I_{\Phi}\left(\frac{x}{\|x\|_{\Phi}}\right)
$$

as $n \rightarrow \infty$ and, by Lemma 2.11, we obtain

$$
I_{\Phi}\left(\frac{x}{\left\|x \chi_{A_{n}}\right\|_{\Phi}}\right) \rightarrow I_{\Phi}\left(\frac{x}{\|x\|_{\Phi}}\right)
$$

as $n \rightarrow \infty$. Since $\Phi\left(t, \frac{x(t) \chi_{A_{n}}(t)}{\left\|x \chi_{A_{n}}\right\|_{\Phi}}\right) \leq \Phi\left(t, \frac{x(t)}{\left\|x \chi_{A_{n}}\right\|_{\Phi}}\right)$ for all $t \in \Omega_{o c}^{0}$, by conditions (2.8) and (2.9), we get

$$
\left\|\Phi\left(t, \frac{x(t)}{\left\|x \chi_{A_{n}}\right\|_{\Phi}}\right)-\Phi\left(t, \frac{x(t) \chi_{A_{n}}(t)}{\left\|x \chi_{A_{n}}\right\|_{\Phi}}\right)\right\|_{L^{1}\left(\Omega_{o c}^{0}\right)}=I_{\Phi}\left(\frac{x}{\left\|x \chi_{A_{n}}\right\|_{\Phi}}\right)-I_{\Phi}\left(\frac{x \chi_{A_{n}}}{\left\|x \chi_{A_{n}}\right\|_{\Phi}}\right) \rightarrow 0
$$


as $n \rightarrow \infty$. Consequently, $\left\|\Phi\left(t, x(t) \chi_{\Omega_{o c}^{0} \backslash A_{n}}\right)\right\|_{L^{1}\left(\Omega_{o c}^{0}\right)} \rightarrow 0$, whence $x \chi_{\Omega_{o c}^{0} \backslash A_{n}} \rightarrow$ 0 as $n \rightarrow \infty$ in measure (because $a_{\Phi}(t)=0$ for all $t \in \Omega_{o c}^{0}$ ). Since the measure $\mu$ is $\sigma$-finite, passing to a subsequence, if necessary, we can assume that $x \chi_{\Omega_{o c}^{0} \backslash A_{n}} \rightarrow 0$ as $n \rightarrow \infty \mu$-a.e. in $\Omega_{o c}^{0}$. Taking any $\lambda>0$, note that

$$
\Phi\left(t, \lambda x(t) \chi_{\Omega_{o c}^{0} \backslash A_{n}}(t)\right) \leq \Phi(t, \lambda x(t)) \in L^{1}\left(\Omega_{o c}\right)
$$

for all $t \in \Omega_{o c}^{0}$ because $x \in E_{+}^{\Phi}(\mu) \backslash\{0\}$. Moreover, $\Phi\left(t, \lambda x(t) \chi_{\Omega_{o c}^{0} \backslash A_{n}}\right) \rightarrow 0$ as $n \rightarrow \infty$ for $\mu$-a.e. $t \in \Omega_{o c}^{0}$. Then, $\left\|\Phi\left(t, \lambda x(t) \chi_{\Omega_{o c}^{0} \backslash A_{n}}\right)\right\|_{L^{1}\left(\Omega_{o c}^{0}\right)} \rightarrow 0$, by the Lebesgue dominated convergence theorem. By the arbitrariness of $\lambda>0$, this means that $\left\|x \chi_{\Omega_{o c}^{0} \backslash A_{n}}\right\|_{\Phi} \rightarrow 0$ as $n \rightarrow \infty$.

Corollary 2.16. The following conditions are equivalent:

(a) $L^{\Phi}(\mu)$ is orthogonally lower locally uniformly monotone,

(b) $L^{\Phi}(\mu)$ is orthogonally strictly monotone,

(c) $a_{\Phi}(t)=0$ for $\mu$-a.e. $t \in \Omega$ and $\Phi \in \Delta_{2}$.

Proof. The implication $(a) \Rightarrow(b)$ is clear. Assume that (b) holds. Then, the condition $\Phi \in \Delta_{2}$ follows from the proof of necessity of Theorem 2.8, where it was proved that if $\Phi \notin \Delta_{2}$, then $L^{\Phi}(\mu)$ is not orthogonally strictly monotone. If $\Phi \in \Delta_{2}$, then $L^{\Phi}(\mu)=E^{\Phi}(\mu)$ and $\operatorname{supp} E^{\Phi}(\mu)=\Omega$. By virtue of Theorem 2.15 , we get that $a_{\Phi}(t)=0$ for $\mu$-a.e. $t \in \Omega$.

Finally, assume that statement (c) holds true. Note that if $\Phi \in \Delta_{2}$, then $b_{\Phi}(t)=\infty$ for $\mu$-a.e. $t \in \Omega$ and $L^{\Phi}(\mu)=E^{\Phi}(\mu)$, so we can apply Theorem 2.15 and the proof is finished.

Theorem 2.17. The following conditions are equivalent:

(a) $L^{\Phi}(\mu)$ is upper locally uniformly monotone,

(b) $\Phi(t,$.$) is strictly increasing on \mathbb{R}_{+}$for $\mu$-a.e. $t \in \Omega$ and $\Phi \in \Delta_{2}$.

Proof. By virtue of Theorem 2.8, implication $(a) \Rightarrow(b)$ is obvious. Now, we will prove that statement (b) implies statement (a). In order to do so, assume that $\Phi(t,$.$) is strictly increasing on \mathbb{R}_{+}$for $\mu$-a.e. $t \in \Omega$ and $\Phi \in \Delta_{2}$. Assume also that $0 \leq x \leq x_{n} \in L^{\Phi}(\mu)$ for any $n \in \mathbb{N}$ and $\lim _{n \rightarrow \infty}\left\|x_{n}\right\|_{\Phi}=\|x\|_{\Phi}$, where $x \neq 0$. Since $\Phi \in \Delta_{2}$, by virtue of statement (3), Corollary 2.2, we get that $I_{\Phi}\left(\frac{x_{n}}{\left\|x_{n}\right\|_{\Phi}}\right)=\left\|x_{n}\right\|_{\Phi}$ for any $n \in \mathbb{N}$ and $I_{\Phi}\left(\frac{x}{\|x\|_{\Phi}}\right)=\|x\|_{\Phi}$, so

$$
I_{\Phi}\left(\frac{x_{n}}{\left\|x_{n}\right\|_{\Phi}}\right)-I_{\Phi}\left(\frac{x}{\|x\|_{\Phi}}\right)=\left\|x_{n}\right\|_{\Phi}-\|x\|_{\Phi} \rightarrow 0
$$

as $n \rightarrow \infty$. Moreover, the assumption that $\Phi \in \Delta_{2}$ implies that $b_{\Phi}(t)=\infty$ for $\mu$-a.e. $t \in \Omega$ and the assumption that $\Phi(t,$.$) is strictly increasing on \mathbb{R}_{+}$implies that $a_{\Phi}(t)=0$ for $\mu$-a.e. $t \in \Omega$. By Theorem 1.9, we get that $E^{\Phi}(\mu)=L^{\Phi}(\mu)$. Applying Lemma 2.11 and the assumption that $\left\|x_{n}\right\|_{\Phi} \rightarrow\|x\|_{\Phi}$ as $n \rightarrow \infty$, we 
obtain that $I_{\Phi}\left(\frac{x}{\left\|x_{n}\right\|_{\Phi}}\right) \rightarrow I_{\Phi}\left(\frac{x}{\|x\|_{\Phi}}\right)=\|x\|_{\Phi}$ as $n \rightarrow \infty$. Consequently,

$$
0 \leq I_{\Phi}\left(\frac{x_{n}}{\left\|x_{n}\right\|_{\Phi}}\right)-I_{\Phi}\left(\frac{x}{\left\|x_{n}\right\|_{\Phi}}\right) \rightarrow 0
$$

as $n \rightarrow \infty$, i.e.

$$
\left\|\Phi\left(t, \frac{x_{n}(t)}{\left\|x_{n}\right\|_{\Phi}}\right)-\Phi\left(t, \frac{x(t)}{\left\|x_{n}\right\|_{\Phi}}\right)\right\|_{L^{1}(\Omega)} \rightarrow 0
$$

as $n \rightarrow \infty$. Hence, by virtue of Lemma 2, p. 97 in [7], there exist a strictly increasing sequence $\left\{n_{k}\right\}_{k=1}^{\infty}$ of natural numbers, a function $y \in L_{+}^{1}(\Omega)$ and a sequence of positive numbers $\left\{\varepsilon_{k}\right\}_{k=1}^{\infty} \downarrow 0$ (we can assume without loss of generality that $\varepsilon_{k} \leq 1$ for any $k \in \mathbb{N}$ ) such that

$$
0 \leq \Phi\left(t, \frac{x_{n_{k}}(t)}{\left\|x_{n_{k}}\right\|_{\Phi}}\right)-\Phi\left(t, \frac{x(t)}{\left\|x_{n_{k}}\right\|_{\Phi}}\right) \leq \varepsilon_{k} y(t) \quad(k \in \mathbb{N}),
$$

for $\mu$-a.e. $t \in \Omega$. Consequently,

$$
\Phi\left(t, \frac{x_{n_{k}}(t)}{\left\|x_{n_{k}}\right\|_{\Phi}}\right) \leq \varepsilon_{k} y(t)+\Phi\left(t, \frac{x(t)}{\left\|x_{n_{k}}\right\|_{\Phi}}\right) \leq y(t)+\Phi\left(t, \frac{x(t)}{\|x\|_{\Phi}}\right)
$$

for $\mu$-a.e. $t \in \Omega$. From inequality (2.10), we conclude (passing to a subsequence, if necessary) that

$$
\Phi\left(t, \frac{x_{n_{k}}(t)}{\left\|x_{n_{k}}\right\|_{\Phi}}\right)-\Phi\left(t, \frac{x(t)}{\left\|x_{n_{k}}\right\|_{\Phi}}\right) \rightarrow 0 \mu-a . e . .
$$

Since $\Phi(t,$.$) is a strictly increasing function for \mu$-a.e. $t \in \Omega$, we conclude that $\frac{x_{n_{k}}}{\left\|x_{n_{k}}\right\|_{\Phi}}-\frac{x}{\left\|x_{n_{k}}\right\|_{\Phi}} \rightarrow 0 \mu$-a.e., whence $\Phi\left(t, \frac{x_{n_{k}}(t)}{\left\|x_{n_{k}}\right\|_{\Phi}}-\frac{x(t)}{\left\|x_{n_{k}}\right\|_{\Phi}}\right) \rightarrow 0 \mu-$ a.e. (and for $\mu$-a.e. $t \in \Omega$ ). Moreover, by (2.11), we get

$$
\Phi\left(t, \frac{x_{n_{k}}(t)}{\left\|x_{n_{k}}\right\|_{\Phi}}-\frac{x(t)}{\left\|x_{n_{k}}\right\|_{\Phi}}\right) \leq \Phi\left(t, \frac{x_{n_{k}}(t)}{\left\|x_{n_{k}}\right\|_{\Phi}}\right) \leq y(t)+\Phi\left(t, \frac{x(t)}{\|x\|_{\Phi}}\right) \in L^{1}(\Omega)
$$

for $\mu$-a.e. $t \in \Omega$. By the order continuity of $L^{1}(\mu)$ as well as the fact that $\left\|x_{n_{k}}\right\|_{\Phi} \leq 2\|x\|_{\Phi}$ for $k$ large enough, we get

$$
0 \leq I_{\Phi}\left(\frac{x_{n_{k}}-x}{2\|x\|_{\Phi}}\right) \leq I_{\Phi}\left(\frac{x_{n_{k}}-x}{\left\|x_{n_{k}}\right\|_{\Phi}}\right) \rightarrow 0
$$

as $k \rightarrow \infty$. Applying the $\Delta_{2}$-condition, we obtain that $I_{\Phi}\left(\lambda\left(x_{n_{k}}-x\right)\right) \rightarrow 0$ as $k \rightarrow \infty$ for any $\lambda>0$, whence $\left\|x_{n_{k}}-x\right\|_{\Phi} \rightarrow 0$ as $k \rightarrow \infty$, by Lemma 2.12. By the double extract subsequence theorem, $\left\|x_{n}-x\right\|_{\Phi} \rightarrow 0$ as $n \rightarrow \infty$ and the proof is finished.

Theorem 2.18. The following conditions are equivalent:

(a) $E^{\Phi}(\mu)$ is upper locally uniformly monotone,

(b) $\mu\left(\Omega_{o c}\right)=0$ or if $\mu\left(\Omega_{o c}\right)>0$, then $\Phi \in \Delta_{2}\left(\Omega_{o c}\right)$ and $\mu\left(\Omega_{n s i}\right)=0$. 
Proof. Assume that statement (b) holds. If $\mu\left(\Omega_{o c}\right)=0$, then $E^{\Phi}(\mu)=\{0\}$ is obviously upper locally uniformly monotone. If $\Phi \in \Delta_{2}\left(\Omega_{o c}\right)$ and $\mu\left(\Omega_{n s i}\right)=0$ under the assumption that $\mu\left(\Omega_{o c}\right)>0$, then Theorems 2.17 and 1.9 yield that $E^{\Phi}(\mu)$ is upper locally uniformly monotone.

Assume that statement (a) holds. Then $\mu\left(\Omega_{o c}\right)=0$ or if $\mu\left(\Omega_{o c}\right)>0$, then $\mu\left(\Omega_{o c} \cap \Omega_{n s i}\right)=0$, by Theorem 2.13 (see also Corollary 2.10). In what follows, we will restrict the measure space $(\Omega, \Sigma, \mu)$ to the measure space $\left(\Omega_{o c},\left.\Sigma\right|_{\Omega_{o c}},\left.\mu\right|_{\Omega_{o c}}\right)$, that is, to the support of $E^{\Phi}(\mu)$. Then $E^{\Phi}(\mu) \neq\{0\}$. Although the beginning of the proof starts similarly to the proof of Lemma 1 on page 65 of the paper [6] by Kamińska (the application of the same technique is enough), we will present it for the convenience of the reader. Suppose that $\Phi \notin \Delta_{2}\left(\Omega_{o c}\right)$.

Note that it is easy to see that the $\Delta_{2}\left(\Omega_{o c}\right)$-condition is satisfied if and only if $\int_{\Omega_{o c}} h_{n}(t) d \mu<\infty$ for some $n \in \mathbb{N}$, where

$$
h_{n}(t)=\sup _{u \in \mathbb{R}_{+}}\left\{\Phi\left(t,\left(1+\frac{1}{n}\right) u\right)-2^{n} \Phi(t, u)\right\} .
$$

Let $\left\{u_{i}\right\}$ be the set of nonnegative rational numbers, $\left\{\Omega_{m}\right\}_{m=1}^{\infty}$ be an ascending sequence of sets such that $\mu\left(\Omega_{m}\right)<\infty$ for any $n \in \mathbb{N}, \mu\left(\Omega_{o c} \backslash \bigcup_{m=1}^{\infty} \Omega_{m}\right)=0$ and

$$
\sup _{t \in \Omega_{m}} \Phi(t, u)<\infty
$$

for every $u \in \mathbb{R}_{+}$and $m \in \mathbb{N}$ (for the fact that such a sequence $\left\{\Omega_{m}\right\}$ exists see [6], p. 64). Let

$$
A_{n m i}=\left\{t \in \Omega_{m}: \Phi\left(t,\left(1+\frac{1}{n}\right) u_{i}\right) \geq 2^{n} \Phi\left(t, u_{i}\right)\right\}
$$

and

$$
g_{n}(t)=\sup _{u \in \mathbb{R}_{+}}\left\{\Phi\left(t,\left(1+\frac{1}{n}\right) u\right): \Phi\left(t,\left(1+\frac{1}{n}\right) u\right) \geq 2^{n} \Phi(t, u)\right\} .
$$

Then,

$$
g_{n}(t)=\sup _{i, m \in \mathbb{N}} \Phi\left(t,\left(1+\frac{1}{n}\right) u_{i} \chi_{A_{n m i}}(t)\right) .
$$

It is obvious that $u_{i} \chi_{A_{n m i}}(t) \in E^{\Phi}(\mu)$ for any $m, i \in \mathbb{N}$. Since $\Phi \notin \Delta_{2}\left(\Omega_{o c}\right)$ then $\int_{\Omega_{o c}} g_{n}(t) d \mu=\infty$ for any $n \in \mathbb{N}$ because $g_{n}(t) \geq h_{n}(t)$ for any $t \in \Omega_{o c}$. Putting

$$
g_{n l}(t)=\max _{1 \leq m, i \leq l} \Phi\left(t,\left(1+\frac{1}{n}\right) u_{i} \chi_{A_{n m i}}(t)\right)
$$


we get that $g_{n l}(t) \uparrow g_{n}(t)$ as $l \rightarrow \infty$ for $\mu$-a.e. $t \in \Omega_{o c}$, whence

$$
\int_{\Omega_{o c}} g_{n l(n)}(t) d \mu \geq 2^{n}
$$

for any $n \in \mathbb{N}$ and some $l(n) \in \mathbb{N}$. Denoting $\hat{x}_{n}(t)=\max _{1 \leq m, i \leq l(n)} u_{i} \chi_{A_{n m i}}(t)$, we obtain that $g_{n l(n)}(t)=\Phi\left(t,\left(1+\frac{1}{n}\right) \hat{x}_{n}(t)\right)$. By Lemma 1.11 together with Remark 1.12 and condition (2.13), we can find a strictly increasing subsequence $\left\{n_{k}\right\}$ in $\mathbb{N}$ and a sequence $\left\{A_{k}\right\}$ of pairwise disjoint sets, chosen in such way that

$$
\mu\left(\Omega_{o c} \backslash \bigcup_{k=1}^{\infty} A_{k}\right)>0
$$

holds, and

$$
\int_{A_{k}} \Phi\left(t,\left(1+\frac{1}{n_{k}}\right) \hat{x}_{n_{k}}(t)\right) d \mu=1
$$

for any $k \in \mathbb{N}$. By the definition of the sets $A_{n m i}$ and functions $\hat{x}_{n}$, we get

$$
\Phi\left(t,\left(1+\frac{1}{n}\right) \hat{x}_{n}(t)\right) \geq 2^{n} \Phi\left(t, \hat{x}_{n}(t)\right)
$$

for any $n \in \mathbb{N}$. Define

$$
y_{k}(t)=\hat{x}_{n_{k}}(t) \chi_{A_{k}}(t)
$$

Then $y_{k} \in E^{\Phi}(\mu)$ and conditions (2.16) and (2.15) yield

$$
\begin{aligned}
I_{\Phi}\left(y_{k}\right) & =I_{\Phi}\left(\hat{x}_{n_{k}} \chi_{A_{k}}\right)=\int_{A_{k}} \Phi\left(t, \hat{x}_{n_{k}}(t)\right) d \mu \\
& \leq \frac{1}{2^{n_{k}}} \int_{A_{k}} \Phi\left(t,\left(1+\frac{1}{n_{k}}\right) \hat{x}_{n_{k}}(t)\right) d \mu=\frac{1}{2^{n_{k}}}
\end{aligned}
$$

for any $k \in \mathbb{N}$. By $(2.15)$, we get

$$
I_{\Phi}\left(\left(1+\frac{1}{n_{k}}\right) y_{k}\right)=\Phi\left(t,\left(1+\frac{1}{n_{k}}\right) \hat{x}_{n_{k}}\right) \mu\left(A_{k}\right)=1
$$

for any $k \in \mathbb{N}$, whence $\left\|\left(1+\frac{1}{n_{k}}\right) y_{k}\right\|_{\Phi} \geq 1$ for any $k \in \mathbb{N}$. Therefore, $\left\|2 y_{k}\right\|_{\Phi} \geq 1$, whence $\left\|y_{k}\right\|_{\Phi} \geq \frac{1}{2}$, by the triangle inequality.

By virtue of the choice of the sequence $\left\{A_{k}\right\}_{k=1}^{\infty}$ satisfying (2.14) as well as condition (2.12), we can find a measurable set $A$ of positive measure such that $A \subset \Omega_{o c} \backslash \bigcup_{k=1}^{\infty} A_{k}$ and $\mu\left(A \cap \Omega_{\widetilde{m}}\right)>0$, where $\Omega_{\widetilde{m}}$ is some set from the sequence of sets $\left\{\Omega_{m}\right\}_{m=1}^{\infty}$ described earlier in the proof (see condition (2.12)). Passing to a subset $B$ of positive measure of the set $A \cap \Omega_{\widetilde{m}}$, if necessary, we can assume that $\mu(B)<\infty$. Take a nonnegative real-valued function $a($. 
such that $\int_{D} \Phi(t, a(t)) d \mu=1$, where $D \in \Sigma, D \subset B, \mu(D)>0$ and define $x(t)=a(t) \chi_{D}(t)$. Then $x \in E^{\Phi}(\mu), I_{\Phi}(x)=1$, whence $\|x\|_{\Phi}=1$. Define

$$
x_{k}=x+y_{k}
$$

for any $k \in \mathbb{N}$. Then $x_{k} \in E^{\Phi}(\mu) \backslash\{0\}, 0 \leq x \leq x_{k}$ and $I_{\Phi}\left(x_{k}\right)=I_{\Phi}(x)+$ $I_{\Phi}\left(y_{k}\right) \leq 1+\frac{1}{2^{n_{k}}}$ for any $k \in \mathbb{N}$. Since $x_{k} \in E^{\Phi}(\mu) \backslash\{0\}$ and $1=\|x\|_{\Phi} \leq\left\|x_{k}\right\|_{\Phi}$ for any $k \in \mathbb{N}$, by statement (2) of Lemma 2.1, we get that $1 \leq\left\|x_{k}\right\|_{\Phi}=$ $I_{\Phi}\left(\frac{x_{k}}{\left\|x_{k}\right\|_{\Phi}}\right) \leq I_{\Phi}\left(x_{k}\right)$ for any $k \in \mathbb{N}$, so

$$
1=\|x\|_{\Phi} \leq\left\|x_{k}\right\|_{\Phi} \leq 1+\frac{1}{2^{n_{k}}}
$$

for any $k \in \mathbb{N}$. Consequently, $\lim _{k \rightarrow \infty}\left\|x_{k}\right\|_{\Phi}=\|x\|_{\Phi}$ and, simultaneously, $\| x_{k}-$ $x\left\|_{\Phi}=\right\| y_{k} \|_{\Phi} \geq \frac{1}{2}$, so $E^{\Phi}(\mu)$ is not, in fact, orthogonally upper locally uniformly monotone, so it is not upper locally uniformly monotone either.

Therefore, the $\Delta_{2}\left(\Omega_{o c}\right)$-condition is necessary for the upper local uniform monotonicity of $E^{\Phi}(\mu)$. Consequently, as it was shown before, $\Omega=\Omega_{o c}$ up to a measure zero set, so statement (b) holds true.

Theorem 2.19. The following conditions are equivalent:

(a) $E^{\Phi}(\mu)$ is orthogonally upper locally uniformly monotone,

(b) $\mu\left(\Omega_{o c}\right)=0$ or if $\mu\left(\Omega_{o c}\right)>0$, then $\Phi \in \Delta_{2}\left(\Omega_{o c}\right)$ and $\mu\left(\operatorname{supp} a_{\Phi}\right)=0$.

Proof. Assume that $E^{\Phi}(\mu)$ is orthogonally upper locally uniformly monotone. Since orthogonal upper local uniform monotonicity implies orthogonal strict monotonicity, by virtue of Theorem 2.15 , we obtain that $\mu\left(\Omega_{o c}\right)=0$ or if $\mu\left(\Omega_{o c}\right)>0$, then $\mu\left(\Omega_{o c} \cap \operatorname{supp} a_{\Phi}\right)=0$. In the case when $\mu\left(\Omega_{o c}\right)>0$, the necessity of the $\Delta_{2}\left(\Omega_{o c}\right)$-condition follows from the proof of Theorem 2.18, implication (a) $\Rightarrow\left(\right.$ b), where it was shown that if $\Phi \notin \Delta_{2}\left(\Omega_{o c}\right)$, then $E^{\Phi}(\mu)$ is not orthogonally upper locally uniformly monotone. Since $\Phi \in \Delta_{2}\left(\Omega_{o c}\right)$, we have that $\mu\left(\Omega \backslash \Omega_{o c}\right)=0$, so statement (b) is true.

Assume that condition (b) holds. If $\mu\left(\Omega_{o c}\right)=0$, then $E^{\Phi}(\mu)=\{0\}$, so the space $E^{\Phi}(\mu)$ is orthogonally upper locally uniformly monotone. If $\mu\left(\Omega_{o c}\right)>0$, then, since $\Phi \in \Delta_{2}\left(\Omega_{o c}\right)$, we get that $\Omega=\Omega_{o c}$ (up to a set of measure zero). Let $x \in E_{+}^{\Phi}(\mu) \backslash\{0\}$ be such that $\mu\left(\operatorname{supp} E^{\Phi}(\mu) \backslash \operatorname{supp} x\right)=\mu\left(\Omega_{o c} \backslash \operatorname{supp} x\right)=$ $\mu(\Omega \backslash \operatorname{supp} x)>0$ and $\left\{x_{n}\right\}_{n=1}^{\infty}$ be a sequence in $E_{+}^{\Phi}(\mu)$ with $\operatorname{supp} x_{n} \subset$ $\Omega \backslash \operatorname{supp} x$ such that $\left\|x+x_{n}\right\|_{\Phi} \rightarrow\|x\|_{\Phi}$ as $n \rightarrow \infty$. We will show that $\left\|x_{n}\right\|_{\Phi} \rightarrow 0$ as $n \rightarrow \infty$. Using the same techniques as those presented in the proof of Theorem 2.13 (conditions (2.3)-(2.5) in the proof), we conclude that $I_{\Phi}\left(\frac{x+x_{n}}{\left\|x+x_{n}\right\|_{\Phi}}\right) \rightarrow I_{\Phi}\left(\frac{x}{\|x\|_{\Phi}}\right)$ and $I_{\Phi}\left(\frac{x}{\left\|x+x_{n}\right\|_{\Phi}}\right) \rightarrow I_{\Phi}\left(\frac{x}{\|x\|_{\Phi}}\right)$, whence 
$I_{\Phi}\left(\frac{x+x_{n}}{\left\|x+x_{n}\right\|_{\Phi}}\right)-I_{\Phi}\left(\frac{x}{\left\|x+x_{n}\right\|_{\Phi}}\right) \rightarrow 0$ as $n \rightarrow \infty$. Since $x_{n}$ and $x$ have orthogonal supports, we obtain

$$
I_{\Phi}\left(\frac{x_{n}}{\left\|x+x_{n}\right\|_{\Phi}}\right)=I_{\Phi}\left(\frac{x+x_{n}}{\left\|x+x_{n}\right\|_{\Phi}}\right)-I_{\Phi}\left(\frac{x}{\left\|x+x_{n}\right\|_{\Phi}}\right) \rightarrow 0
$$

as $n \rightarrow \infty$. Next, similarly as in the proof of Theorem 2.17 , we can find a subsequence $\left(x_{n_{k}}\right)$ of $\left(x_{n}\right)$, a sequence $\left(\varepsilon_{k}\right)$ with $0<\varepsilon_{k} \rightarrow 0$ as $k \rightarrow \infty$ and $y \in L_{+}^{1}(\Omega)$ such that $\Phi\left(t, \frac{x_{n_{k}}(t)}{2\|x\|_{\Phi}}\right) \leq \Phi\left(t, \frac{x_{n_{k}}(t)}{\left\|x+x_{n_{k}}\right\|_{\Phi}}\right) \leq \varepsilon_{k} y$ for $k$ large enough and all $t \in \Omega$. Since $\varepsilon_{k} \rightarrow 0$ as $k \rightarrow \infty$, we obtain that $I_{\Phi}\left(\frac{x_{n_{k}}}{2\|x\|_{\Phi}}\right) \rightarrow 0$ as $k \rightarrow \infty$. Applying the $\Delta_{2}\left(\Omega_{o c}\right)$-condition, we get that $I_{\Phi}\left(\lambda x_{n_{k}}\right) \rightarrow 0$ as $k \rightarrow \infty$ for any $\lambda>0$, whence $\left\|x_{n_{k}}\right\|_{\Phi} \rightarrow 0$ as $k \rightarrow \infty$. The double extract subsequence theorem finishes the proof.

Corollary 2.20. The following conditions are equivalent:

(a) $L^{\Phi}(\mu)$ is orthogonally upper locally uniformly monotone,

(b) $L^{\Phi}(\mu)$ is orthogonally strictly monotone,

(c) $\mu\left(\operatorname{supp} a_{\Phi}\right)=0$ and $\Phi \in \Delta_{2}$.

Proof. It is obvious that (a) implies (b). Implication (b) $\Rightarrow$ (c) follows from Corollary 2.16. Assume that statement (c) holds true. Since $\Phi \in \Delta_{2}$ and $a_{\Phi}(t)=0$ for $\mu$-a.e. $t \in \Omega$ then $E^{\Phi}(\mu)=L^{\Phi}(\mu)$, so it is enough to apply Theorem 2.19.

Open Access. This article is distributed under the terms of the Creative Commons Attribution 4.0 International License (http://creativecommons.org/licenses/by/4.0/), which permits unrestricted use, distribution, and reproduction in any medium, provided you give appropriate credit to the original author(s) and the source, provide a link to the Creative Commons license, and indicate if changes were made.

Publisher's Note Springer Nature remains neutral with regard to jurisdictional claims in published maps and institutional affiliations.

\section{References}

[1] Birkhoff, G.: Lattice Theory. Providence, Providence (1967)

[2] Chen, S.: Geometry of Orlicz spaces. Diss. Math. 356, 1-204 (1996)

[3] Cui, Y., Hudzik, H., Kaczmarek, R., Kolwicz, P.: Geometric properties of F-normed Orlicz spaces. Aequ. Math. 93, 311-343 (2019)

[4] Hudzik, H.: Strict convexity of Musielak-Orlicz spaces with Luxemburg's norm. Bull. Acad. Polon. Sci. Sér. Sci. Math. 29(5-6), 235-247 (1981)

[5] Hudzik, H., Kaczmarek, R., Wójtowicz, M.: Some monotonicity properties in certain snormed $(0<s<1)$ and $F$-normed lattices. J. Nonlinear Convex Anal. 17(10), 1985-2011 (2016). + Corrigendum in J. Nonlinear Convex Anal. 18(12), 2275 (2017)

[6] Kamińska, A.: Some convexity properties of Musielak-Orlicz spaces of Bochner type. In: Proceedings of the 13th Winter School on Abstract Analysis (Srní, 1985). Rend. Circ. Mat. Palermo 10(2), 63-73 (1986) 
[7] Kantorovich, L.V., Akilov, G.P.: Functional Analysis, 2nd edn. Pergamon Press, Oxford (1982). (English translation)

[8] Kozek, A.: Convex integral functionals on Orlicz spaces. Comment. Math. Prace Mat. 21(1), 109-135 (1980)

[9] Mazur, S., Orlicz, W.: On some classes of linear spaces. Stud. Math. 17, 97-119 (1958). In: Reprinted W. Orlicz, Collected Papers, PWN, Warszawa, pp. 981-1003 (1988)

[10] Musielak, J.: Orlicz Spaces and Modular Spaces. In: Lecture Notes Math., vol. 1034. Springer, Berlin (1983)

[11] Musielak, J., Orlicz, W.: On modular spaces. Stud. Math. 18, 49-65 (1959)

Radosław Kaczmarek

Faculty of Mathematics and Computer Science

Adam Mickiewicz University, Poznań

Uniwersytetu Poznańskiego 4

61-614 Poznan

Poland

e-mail: radekk@amu.edu.pl

Received: July 23, 2019

Revised: October 26, 2019 